\title{
Possibility Method for Triangular Intuitionistic Fuzzy Multi-attribute Group Decision Making with Incomplete Weight Information
}

\author{
Shu-Ping Wan ${ }^{\text {a,b }}$ Jiu-Ying Dong ${ }^{c}$ \\ ${ }^{a}$ College of Information Technology, Jiangxi University of Finance and Economics, \\ Nanchang, Jiangxi 330013, China \\ bJiangxi Key Laboratory of Data and Knowledge Engineering, Jiangxi University of Finance and Economics, \\ Nanchang 330013 \\ ${ }^{c}$ College of Statistics, Jiangxi University of Finance and Economics, \\ Nanchang, Jiangxi 330013, China
}

E-mail: shupingwan@163.com

Received 24 April 2012

Accepted 26 May 2013

\begin{abstract}
Triangular intuitionistic fuzzy numbers (TIFNs) are a special kind of intuitionistic fuzzy sets (IFSs) on a real number set. TIFNs are useful to deal with ill-known quantities in decision data and decision making problems themselves. The purpose of this paper is on developing a new ranking method of TIFNs and application to multiattribute group decision making (MAGDM) problems in which the attribute values are expressed with TIFNs and the information on attribute weights is incomplete. The weighted average operator of TIFNs is defined, the concepts of the possibility mean, variance of TIFNs as well as standard deviation are introduced. Hereby two new ranking indices considering the risk attitude of decision maker are developed to rank TIFNs. In the proposed group decision method, the collective overall attribute values of alternatives are obtained by using the weighted average operator of TIFNs We construct the multi-objective programming of maximizing the ranking indices of membership and non-membership functions on alternative's collective overall attribute values, which is transformed into a single linear programming model by using the membership function based weighted sum approach. Thus, the ranking indices of membership and non-membership functions for the alternatives are derived, which are used to rank the alternatives. A personnel selection example is analyzed to demonstrate the applicability, universality and flexibility of the proposed models and method.
\end{abstract}

Keywords: Multi-attribute group decision making; Triangular intuitionistic fuzzy number; Possibility mean; Possibility variance; Possibility standard deviation 


\section{Introduction}

The intuitionistic fuzzy set (IFS) introduced by Atanassov ${ }^{1}$ is a generalization of the fuzzy set (FS) $\left(\right.$ Zadeh $\left.^{2}\right)$. The IFS may describe more abundant and flexible information than the FS when uncertain information is involved. The notable feature of IFS is to assign each element a membership degree and a nonmembership degree. The IFS is useful to deal with uncertainty and vagueness. Atanassov and Gargov ${ }^{3}$ further generalized the IFS in the spirit of the ordinary interval-valued fuzzy set (IVFS) and defined the notion of an interval-valued intuitionistic fuzzy set (IVIFS). The IFS and IVIFS have been widely studied, developed, and applied to various fields, such as multiattribute decision making (MADM), multi-attribute group decision making (MAGDM), logic reasoning, pattern recognition.

Fuzzy numbers are a special case of fuzzy sets. Similarly, intuitionistic fuzzy number (IFN) is a special IFS defined on real number set. As a generalization of fuzzy numbers (Dubois and Prade ${ }^{4}$ ), an IFN seems to suitably describe an ill-known quantity $\left(\mathrm{Li}^{5}\right)$. The triangular IFN (TIFN) is a typical IFN, which has been received a little attention and made some progresses in the definition and ranking method. For example, Shu et al. ${ }^{6}$ defined a triangular IFN (TIFN) in a similar way to the fuzzy number introduced by (Dubois and Prade ${ }^{4}$ ) and developed an algorithm for intuitionistic fuzzy fault tree analysis. $\mathrm{Li}^{5}$ pointed out and corrected some errors in the definition of the four arithmetic operations for the TIFNs (Shu et $\mathrm{al}^{6}{ }^{6}$ ). $\mathrm{Li}^{7}$ analyzed the concept of the TIFN in detail and defined the value index and ambiguity index. Then, $\mathrm{He}$ proposed the ranking method based on the ratio of the value index to the ambiguity index and applied to the MADM problems in which the ratings of alternatives on attributes are expressed using TIFNs. Li et al. ${ }^{8}$ developed a value and ambiguity based method to rank TIFNs and applied to solve MADM with TIFNs. Nan et al. ${ }^{9}$ defined the ranking order relations of TIFNs and investigated the fuzzy matrix games with payoffs of TIFNs.

The possibility theory of FSs was proposed by Zadeh $^{10}$. Its academic meaning is in building a theoretical framework of real applications for FSs. Dubois and Prade ${ }^{11}$ defined the notion of expectation for fuzzy numbers of intervals. This expectation is intervalvalued and remains additive in the sense of fuzzy interval addition. Carlsson and Fullér ${ }^{12}$ introduced the notations of lower and upper possibilistic mean values and the interval-valued possibilistic mean. They also introduced the notations of crisp possibilistic mean value and crisp possibilistic variance of continuous possibility distributions. Introducing a weighting function measuring importance of $\gamma$-level sets of fuzzy numbers, Fullér and Majlender ${ }^{13}$ defined the weighted lower and upper possibilistic mean values, crisp possibilistic mean value and variance of fuzzy numbers. Saeidifar and Pasha $^{14}$ investigated the possibilistic moments of fuzzy numbers and their applications. Chen and $\operatorname{Tan}^{15}$ introduced the definitions of the possibilistic mean, variance and covariance of multiplication of fuzzy numbers, and showed some properties of these definitions.

In statistics, central tendency and distribution dispersion are considered to be the important measures. For fuzzy numbers, two of the most useful measures are the mean and variance of fuzzy numbers. The possibility mean and variance are the important mathematical characteristics of fuzzy numbers. The possibilistic mean, variance and covariance of fuzzy numbers, defined by Carlsson and Fullér ${ }^{12}$, were used to solve many real world problems. For example, Carlsson et al. ${ }^{16}$ and Jana et al. ${ }^{17}$ applied possibilistic mean value and variance to solve Markowitz mean-variance portfolio selection problem under the assumption that the returns of assets were fuzzy numbers. Zhang and $\mathrm{Xiao}^{18}$ applied weighted possibilistic mean and variance of fuzzy numbers to the decision making. The possibility mean of a TIFN can be used to measure the value of the TIFN, while the possibility variance of a TIFN is able to describe the uncertainty and vagueness of the TIFN. Therefore, defining the possibility mean and variance of TIFNs is of a great importance for scientific researches and real applications. Wan and $\mathrm{Li}^{19}$ firstly defined the possibility mean, variance and standard deviation of TIFNs and developed a ranking method based on the ratio of the possibility mean to 
standard deviation and applied to MADM with TIFNs. Wan et al. ${ }^{20}$ defined the weighted possibility means of TIFNs and proposed the extended VIKOR method to MAGDM with TIFNs. Nevertheless, the existing ranking methods of TIFNs $\left(\mathrm{Li}^{7}\right.$; Li et al. ${ }^{8}$; Nan et al. ${ }^{9}$; Wan and $\mathrm{Li}^{19}$ ) ignored the risk attitudes of decision makers (DMs). During the process of decision making, difference DMs have different risk preferences. It is necessary to introduce the risk attitudes to rank TIFNs. There is no such investigation as far as we know.

With ever increasing complexity of modern society, continued expansion of the scale and the diversification of business, lots of important decision problems need more and more decision makers (DMs) to participate the decision making. The group decision analysis emerges as the times require and becomes an important research field of management science. In the multi-attribute group decision making (MAGDM), key information includes ratings (or values) of alternatives on attributes and attribute weights (or preference information). Preference information given by the DMs reflects importance of attributes to the overall MAGDM problems. With ever increasing complexity in many real decision situations, there are often some challenges for the DMs to provide precise and complete preference information due to time pressure, lack of knowledge (or data) and their limited expertise about the problem domain. In other words, usually weights are totally unknown or partially known a priori. Namely, weight preference information in MAGDM problems is usually incomplete. Recently, there are some methods for solving MADM problems with incomplete preference information (Herrera-Viedma ${ }^{21}$; Podinovski ${ }^{22}$; Wang et al. ${ }^{23} ; \mathrm{Li}^{24}$; Wan and $\mathrm{Li}^{25}$; $\mathrm{Li}$ and $\mathrm{Wan}^{26}$ ). However, due to the fact that the IFS has two independent and conflicting functions which are membership and nonmembership degrees, most of them are not applicable to the MADM or MAGDM problems in which ratings of alternatives on attributes are expressed with IFSs (especially TIFNs) and weight information is incomplete. Hence, the aim of this paper is to introduce the risk attitude to develop a new ranking method for TIFNs and further apply to the MAGDM problems with TIFNs and incomplete weight preference information.

The remainder of this paper is structured as follows. Section 2 introduces the definition, operation laws, and weighted average operator of TIFNs. Section 3 gives the concepts of the possibility mean, variance of
TIFNs as well as standard deviation and thereby develops a new ranking method of TIFNs. Section 4 constructs the MAGDM model using TIFNs with incomplete weight information and proposes the corresponding group decision method. A personnel selection example and comparison analysis are given in Section 5. Section 6 ends the paper with some conclusions.

\section{Definition and operation laws and weighted average operator of triangular intuitionistic fuzzy numbers}

In this section, we briefly review the definition, operation laws of TIFNs and define the weighted average operator of TIFNs.

\subsection{Definition of TIFN}

Definition $1\left(\mathrm{Li}^{7}\right)$. A TIFN $\tilde{a}=\left((\underline{a}, a, \bar{a}) ; w_{\tilde{a}}, u_{\tilde{a}}\right)$ is a special kind of intuitionistic fuzzy set on a real number set $R$, whose membership function and nonmembership function are defined as follows:

and

$$
\mu_{\tilde{a}}(x)=\left\{\begin{array}{l}
\frac{x-\underline{a}}{a-\underline{a}} w_{\tilde{a}}, \text { if } \underline{a} \leq x<a \\
w_{\tilde{a}}, \text { if } x=a \\
\bar{a}-x \\
\bar{a}-a \\
0, \text { if } x<\underline{a} \text { or } x>\bar{a}
\end{array}\right.
$$

$$
v_{\tilde{a}}(x)=\left\{\begin{array}{l}
\frac{a-x+(x-\underline{a}) u_{\tilde{a}}}{a-\underline{a}}, \text { if } \underline{a} \leq x<a \\
\frac{u_{\tilde{a}}, \text { if } x=a}{x-a+(\bar{a}-x) u_{\tilde{a}}}, \text { if } a<x \leq \bar{a} \\
\bar{a}-a
\end{array},\right.
$$

respectively, depicted as in Fig. 1. The values $w_{\tilde{a}}$ and $u_{\tilde{a}}$ represent the maximum degree of membership and the minimum degree of non-membership, respectively, such that they satisfy the conditions: $0 \leq w_{\tilde{a}} \leq 1$, $0 \leq u_{\tilde{a}} \leq 1$ and $w_{\tilde{a}}+u_{\tilde{a}} \leq 1$. Let $\pi_{\tilde{a}}(x)=1-\mu_{\tilde{a}}(x)-v_{\tilde{a}}(x)$, which is called an intuitionistic fuzzy index of an element $x$ in $\tilde{a}$.

A TIFN $\tilde{a}=\left((\underline{a}, a, \bar{a}) ; w_{\tilde{a}}, u_{\tilde{a}}\right)$ may express an illknown quantity "approximate $a$ ", which is approximately equal to $a$. Namely, the ill-known quantity "approximate $a$ " is expressed using any value between $\underline{a}$ and $\bar{a}$ with different degrees of membership and degrees of non-membership. In other 
words, the most possible value is $a$ with the degree $w_{\tilde{a}}$ of membership and the degree $u_{\tilde{a}}$ of non-membership; the pessimistic value is $\underline{a}$ with the degree 0 of membership and the degree 1 of non- membership; the optimistic value is $\bar{a}$ with the degree 0 of membership and the degree 1 of non-membership; other values are any $x \in(\underline{a}, \bar{a})$ with the degree $\mu_{\tilde{a}}(x)$ of membership and the degree $v_{\tilde{a}}(x)$ of non-membership $\left(\mathrm{Li}^{7}\right)$.

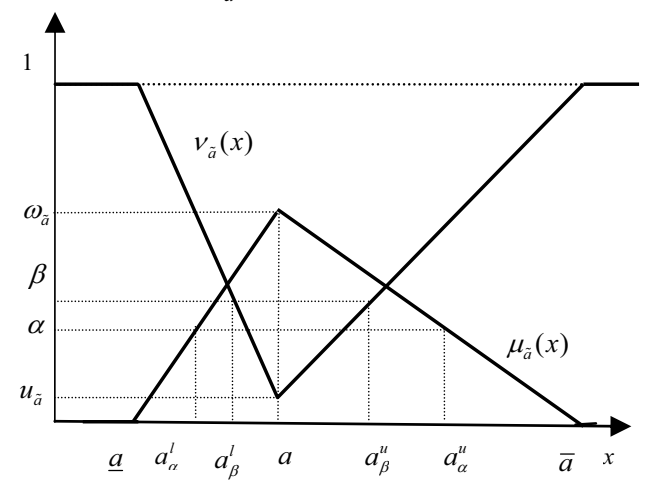

Fig. $1 \alpha$-cut set of membership function and $\beta$-cut set of nonmembership function

It is easily seen that $\mu_{\tilde{a}}(x)+v_{\tilde{a}}(x)=1$ for any $x \in R$. If $w_{\tilde{a}}=1$ and $u_{\tilde{a}}=0$, the TIFN $\tilde{a}=\left((\underline{a}, a, \bar{a}) ; w_{\tilde{a}}, u_{\tilde{a}}\right)$ degenerates to $\tilde{a}=((\underline{a}, a, \bar{a}) ; 1,0)$, which is just a triangular fuzzy number $(\mathrm{TFN}) \tilde{a}=(\underline{a}, a, \bar{a})$. Therefore, the concept of the TIFN is a generalization of that of the TFN. Two new parameters $w_{\tilde{a}}$ and $u_{\tilde{a}}$ are introduced to reflect the confidence and non-confidence levels of the TIFN, respectively. Compared with the TFN, a TIFN may express more uncertainty $\left(\mathrm{Li}^{7}\right)$.

If $\underline{a} \geq 0$ and one of the three values $\underline{a}, a$ and $\bar{a}$ is not equal to 0 , then the $\operatorname{TIFN} \tilde{a}=\left((\underline{a}, a, \bar{a}) ; w_{\tilde{a}}, u_{\tilde{a}}\right)$ is called a positive TIFN, denoted by $\tilde{a}>0$. Likewise, if $\bar{a} \leq 0$ and one of the three values $\underline{a}, a$ and $\bar{a}$ is not equal to 0 , then the TIFN $\tilde{a}=\left((\underline{a}, a, \bar{a}) ; w_{\tilde{a}}, u_{\tilde{a}}\right)$ is called a negative TIFN, denoted by $\tilde{a}<0\left(\mathrm{Li}^{7}\right)$.

\subsection{Operation laws and weighted average operator for the TIFNS}

Definition $2\left(\mathrm{Li}^{7}\right)$. Let $\tilde{a}=\left((\underline{a}, a, \bar{a}) ; w_{\tilde{a}}, u_{\tilde{a}}\right)$ and $\tilde{b}=\left((\underline{b}, b, \bar{b}) ; w_{\tilde{b}}, u_{\tilde{b}}\right)$ be two TIFNs and $\lambda$ be a real number. Then the operation laws for TIFNs are defined as follows:

1) $\tilde{a}+\tilde{b}=\left((\underline{a}+\underline{b}, a+b, \bar{a}+\bar{b}) ; w_{\tilde{a}} \wedge w_{\tilde{b}}, u_{\tilde{a}} \vee u_{\tilde{b}}\right)$, where the symbols " $\wedge$ " and " $\vee$ " mean min and max operators, respectively;
2) $\lambda \tilde{a}=\left\{\begin{array}{l}\left((\lambda \underline{a}, \lambda a, \lambda \bar{a}) ; w_{\tilde{a}}, u_{\tilde{a}}\right), \text { if } \lambda>0 \\ \left((\lambda \bar{a}, \lambda a, \lambda \underline{a}) ; w_{\tilde{a}}, u_{\tilde{a}}\right), \text { if } \lambda<0\end{array}\right.$.

Definition 3. Assume that $\tilde{a}_{j} \quad(j=1,2, \cdots, n)$ is a collection of the TIFNs. Let $\varphi_{\omega}: M^{n} \rightarrow M$. If

$$
\varphi_{\omega}\left(\tilde{a}_{1}, \tilde{a}_{2}, \cdots, \tilde{a}_{n}\right)=\sum_{j=1}^{n} \omega_{j} \tilde{a}_{j},
$$

where $M$ is the set of all TIFNs, $\omega=\left(\omega_{1}, \omega_{2}, \cdots, \omega_{n}\right)^{\mathrm{T}}$ is the weight vector of $\tilde{a}_{j}(j=1,2, \cdots, n)$, satisfying that $0 \leq \omega_{j} \leq 1 \quad(j=1,2, \cdots, n)$ and $\sum_{j=1}^{n} \omega_{j}=1$, then the function $\varphi_{\omega}$ is called the $n$-dimensional weighted average operator for the TIFNs. Especially, if $\omega_{j}=1 / n$ $(j=1,2, \cdots, n)$, then the $\varphi_{\omega}$ operator is reduced to the arithmetic average operator for the TIFNs.

Theorem 1. Let $\tilde{a}_{j}(j=1,2, \cdots, n)$ be a collection of the TIFNs. Their aggregated value by using $\varphi_{\omega}$ operator is also an TIFN, and

$$
\begin{gathered}
\varphi_{\omega}\left(\tilde{a}_{1}, \tilde{a}_{2}, \cdots, \tilde{a}_{n}\right)=\left(\left(\sum_{j=1}^{n} \omega_{j} \underline{a}_{j}, \sum_{j=1}^{n} \omega_{j} a_{j}, \sum_{j=1}^{n} \omega_{j} \bar{a}_{j}\right) ;\right. \\
\bigwedge_{1 \leq j \leq n}\left\{w_{\tilde{a}_{j}}\right\},{\left.\underset{1 \leq j \leq n}{\bigvee}\left\{u_{\tilde{a}_{j}}\right\}\right) .}^{.}
\end{gathered}
$$

Proof of Theorem 1 is easily completed according to Definition 2 (omitted).

\section{A new ranking method of TIFNs considering the risk attitude of $\mathrm{DM}$}

For the purpose of making this paper smoother and easier to understand, some concepts, such as possibility mean, variance, and standard deviation of TIFN (Wan and $\mathrm{Li}^{19}$ ), are introduced in this section. Hereby, a new ranking method of TIFNs is proposed considering the risk attitude of DM.

\subsection{The possibility mean of a TIFN}

Definition $4 \quad\left(\mathrm{Li}^{7}\right)$. A $\quad(\alpha, \beta) \quad$-cut set of $\tilde{a}=\left((\underline{a}, a, \bar{a}) ; w_{\tilde{a}}, u_{\tilde{a}}\right)$ is a crisp subset of $R$, which is defined as follows:

$$
\tilde{a}_{\alpha, \beta}=\left\{x \mid \mu_{\tilde{a}}(x) \geq \alpha, v_{\tilde{a}}(x) \leq \beta\right\},
$$

where $0 \leq \alpha \leq w_{\tilde{a}}, u_{\tilde{a}} \leq \beta \leq 1$ and $0 \leq \alpha+\beta \leq 1$.

Definition $5\left(\mathrm{Li}^{7}\right)$. A $\alpha$-cut set of $\tilde{a}=\left((\underline{a}, a, \bar{a}) ; w_{\tilde{a}}, u_{\tilde{a}}\right)$ is a crisp subset of $R$, which is defined as follows:

$$
\tilde{a}_{\alpha}=\left\{x \mid \mu_{\tilde{a}}(x) \geq \alpha\right\} \text {. }
$$

Definition $6\left(\mathrm{Li}^{7}\right)$. A $\beta$-cut set of $\tilde{a}=\left((\underline{a}, a, \bar{a}) ; w_{\tilde{a}}, u_{\tilde{a}}\right)$ is a crisp subset of $R$, which is defined as follows:

$$
\tilde{a}_{\beta}=\left\{x \mid v_{\tilde{a}}(x) \leq \beta\right\} \text {. }
$$


It directly follows from Fig. 1 and Definitions 1, 5 and 6 that

$$
\tilde{a}_{\alpha}=\left[a_{\alpha}^{l}, a_{\alpha}^{u}\right]=\left[\underline{a}+\frac{(a-\underline{a}) \alpha}{w_{\tilde{a}}}, \bar{a}-\frac{(\bar{a}-a) \alpha}{w_{\tilde{a}}}\right],
$$

and

$$
\tilde{a}_{\beta}=\left[a_{\beta}^{l}, a_{\beta}^{u}\right]=\left[\frac{(1-\beta) a+\left(\beta-u_{\tilde{a}}\right) \underline{a}}{1-u_{\tilde{a}}}, \frac{(1-\beta) a+\left(\beta-u_{\tilde{a}}\right) \bar{a}}{1-u_{\tilde{a}}}\right] .
$$

Motivated by Fullér and Majlender ${ }^{13}$, Wan and $\mathrm{Li}^{19}$ gave the definitions of the possibility means of TIFNs as follows.

Definition 7. Let $\tilde{a}_{\alpha}=\left[a_{\alpha}^{l}, a_{\alpha}^{u}\right]$ be the $\alpha$-cut set of a TIFN $\tilde{a}=\left((\underline{a}, a, \bar{a}) ; w_{\tilde{a}}, u_{\tilde{a}}\right)$ with $0 \leq \alpha \leq w_{\tilde{a}}$. The lower and upper possibility means of membership function for the TIFN $\tilde{a}=\left((\underline{a}, a, \bar{a}) ; w_{\tilde{a}}, u_{\tilde{a}}\right)$ are, respectively, defined as follows:

$$
\underline{m}_{\mu}(\tilde{a})=2 \int_{0}^{w_{\tilde{a}}} \operatorname{Pos}\left[\tilde{a} \leq a_{\alpha}^{l}\right] a_{\alpha}^{l} \mathrm{~d} \alpha
$$

and

$$
\bar{m}_{\mu}(\tilde{a})=2 \int_{0}^{w_{\tilde{a}}} \operatorname{Pos}\left[\tilde{a} \geq a_{\alpha}^{u}\right] a_{\alpha}^{u} \mathrm{~d} \alpha,
$$

where Pos means possibility (Fullér and Majlender ${ }^{13}$ ) and

$$
\begin{aligned}
& \operatorname{Pos}\left[\tilde{a} \leq a_{\alpha}^{l}\right]=\sup _{x \leq a_{\alpha}^{l}} \mu_{\tilde{a}}(x)=\alpha, \\
& \operatorname{Pos}\left[\tilde{a} \geq a_{\alpha}^{u}\right]=\sup _{x \geq a_{\alpha}^{u}} \mu_{\tilde{a}}(x)=\alpha .
\end{aligned}
$$

It is apparent that the lower possibility mean $\underline{m}_{\alpha}(\tilde{a})$ of membership function is nothing else but the lower possibility weighted average of the minimum of the $\alpha$-cut set. The upper possibility mean $\bar{m}_{\alpha}(\tilde{a})$ of membership function is nothing else but the upper possibility weighted average of the maximum of the $\alpha$ cut set.

Definition 8. Let $\tilde{a}_{\beta}=\left[a_{\beta}^{l}, a_{\beta}^{u}\right]$ be the $\beta$-cut set of a TIFN $\tilde{a}=\left((\underline{a}, a, \bar{a}) ; w_{\tilde{a}}, u_{\tilde{a}}\right)$ with $u_{\tilde{a}} \leq \beta \leq 1$. The lower and upper possibility means of non-membership function for the TIFN $\tilde{a}=\left((\underline{a}, a, \bar{a}) ; w_{\tilde{a}}, u_{\tilde{a}}\right)$ are, respectively, defined as follows:

$$
\underline{m}_{v}(\tilde{a})=2 \int_{u_{\tilde{a}}}^{1} \operatorname{Pos}\left[\tilde{a} \leq a_{\beta}^{l}\right] a_{\beta}^{l} \mathrm{~d} \beta
$$

and

$$
\bar{m}_{v}(\tilde{a})=2 \int_{u_{\bar{a}}}^{1} \operatorname{Pos}\left[\tilde{a} \geq a_{\beta}^{u}\right] a_{\beta}^{u} \mathrm{~d} \beta,
$$

where

$$
\begin{gathered}
\operatorname{Pos}\left[\tilde{a} \leq \tilde{a}_{\beta}^{l}\right]=\sup _{x \leq a_{\beta}^{l}} v_{\tilde{a}}(x)=\beta, \\
\operatorname{Pos}\left[\tilde{a} \geq a_{\beta}^{u}\right]=\sup _{x \geq a_{\beta}^{u}} v_{\tilde{a}}(x)=\beta .
\end{gathered}
$$

Analogously, the lower possibility mean of nonmembership function is nothing else but the lower possibility weighted average of the minimum of the $\beta$ cut set. The upper possibility mean of non-membership function is nothing else but the upper possibility weighted average of the maximum of the $\beta$-cut set.

Definition 9. For a TIFN $\tilde{a}=\left((\underline{a}, a, \bar{a}) ; w_{\tilde{a}}, u_{\tilde{a}}\right)$, the possibility means of membership and non-membership functions are, respectively, defined as follows:

$$
m_{\mu}(\tilde{a})=\frac{1}{2}\left[\underline{m}_{\mu}(\tilde{a})+\bar{m}_{\mu}(\tilde{a})\right]
$$

and

$$
m_{v}(\tilde{a})=\frac{1}{2}\left[\underline{m}_{v}(\tilde{a})+\bar{m}_{v}(\tilde{a})\right] .
$$

Obviously, $m_{\mu}(\tilde{a})$ synthetically reflects the information on every membership degree, and may be regarded as a central value that represents from the membership function point of view. Likewise, $m_{v}(\tilde{a})$ synthetically reflects the information on every nonmembership degree, and may be regarded as a central value that represents from the non-membership function point of view.

According to Eqs. (3)-(6), (9) and (10), we have

$$
\begin{gathered}
\underline{m}_{\mu}(\tilde{a})=\frac{1}{3}(\underline{a}+2 a) w_{\tilde{a}}^{2}, \\
\bar{m}_{\mu}(\tilde{a})=\frac{1}{3}(\bar{a}+2 a) w_{\tilde{a}}^{2}, \\
\underline{m}_{v}(\tilde{a})=\left(a-\underline{a}_{\tilde{a}}\right)\left(1+u_{\tilde{a}}\right)-\frac{2}{3}(a-\underline{a})\left(1+u_{\tilde{a}}+u_{\tilde{a}}{ }^{2}\right), \\
\bar{m}_{v}(\tilde{a})=\left(a-\bar{a} u_{\tilde{a}}\right)\left(1+u_{\tilde{a}}\right)+\frac{2}{3}(\bar{a}-a)\left(1+u_{\tilde{a}}+u_{\tilde{a}}{ }^{2}\right) .
\end{gathered}
$$

Further, by Eqs. (13) and (14), we have

$$
\begin{gathered}
m_{\mu}(\tilde{a})=\frac{1}{6}(\underline{a}+4 a+\bar{a}) w_{\tilde{a}}^{2}, \\
m_{v}(\tilde{a})=\frac{1}{6}\left(1-u_{\tilde{a}}\right)\left[2\left(1+2 u_{\tilde{a}}\right) a+\left(2+u_{\tilde{a}}\right)(\underline{a}+\bar{a})\right],
\end{gathered}
$$

\subsection{The possibility variance and standard deviation of a TIFN}

Wan and $\mathrm{Li}^{19}$ also defined the possibility variances and standard deviations of a TIFN as follows.

Definition 10. For a TIFN $\tilde{a}=\left((\underline{a}, a, \bar{a}) ; w_{\tilde{a}}, u_{\tilde{a}}\right)$, the possibility variance of membership function is defined as follows:

$$
\begin{aligned}
V_{\mu}(\tilde{a})= & \int_{0}^{w_{\tilde{a}}} \operatorname{Pos}\left[\tilde{a} \leq a_{\alpha}^{l}\right]\left[\frac{1}{2}\left(a_{\alpha}^{l}+a_{\alpha}^{u}\right)-a_{\alpha}^{l}\right]^{2} \mathrm{~d} \alpha \\
& +\int_{0}^{w_{\tilde{a}}} \operatorname{Pos}\left[\tilde{a} \geq a_{\alpha}^{u}\right]\left[\frac{1}{2}\left(a_{\alpha}^{l}+a_{\alpha}^{u}\right)-a_{\alpha}^{u}\right]^{2} \mathrm{~d} \alpha \\
= & 2 \int_{0}^{w_{\tilde{a}}}\left(\frac{a_{\alpha}^{u}-a_{\alpha}^{l}}{2}\right)^{2} \alpha \mathrm{d} \alpha=\frac{1}{24}(\bar{a}-\underline{a})^{2} w_{\tilde{a}}^{2} .
\end{aligned}
$$

Definition 11. For a TIFN $\tilde{a}=\left((\underline{a}, a, \bar{a}) ; w_{\tilde{a}}, u_{\tilde{a}}\right)$, the possibility variance of non-membership function is defined as follows:

$V_{v}(\tilde{a})=\int_{u_{\tilde{a}}}^{1} \operatorname{Pos}\left[\tilde{a} \leq a_{\beta}^{l}\right]\left[\frac{1}{2}\left(a_{\beta}^{l}+a_{\beta}^{u}\right)-a_{\beta}^{l}\right]^{2} \mathrm{~d} \beta$ 


$$
\begin{gathered}
+\int_{u_{\bar{a}}}^{1} \operatorname{Pos}\left[\tilde{a} \geq a_{\beta}^{u}\right]\left[\frac{1}{2}\left(a_{\beta}^{l}+a_{\beta}^{u}\right)-a_{\beta}^{u}\right]^{2} \mathrm{~d} \beta \\
=2 \int_{u_{\tilde{a}}}^{1}\left(\frac{a_{\beta}^{u}-a_{\beta}^{l}}{2}\right)^{2} \beta \mathrm{d} \beta=\frac{1}{24}\left(3+u_{\tilde{a}}\right)\left(1-u_{\tilde{a}}\right)(\bar{a}-\underline{a})^{2} .
\end{gathered}
$$

The possibility variance of membership function is defined as the expected value of the squared deviations between the arithmetic mean and the endpoints of $\alpha$ cut set, i.e., the lower possibility-weighted average of the squared distance between the left-hand endpoint and the arithmetic mean of the endpoints of $\alpha$-cut set plus the upper possibility-weighted average of the squared distance between the right-hand endpoint and the arithmetic mean of the endpoints of $\alpha$-cut set. The possibility variance of non-membership function is explained similarly.

It is easily seen that $a_{\alpha}^{u}-a_{\alpha}^{l}$ and $a_{\beta}^{u}-a_{\beta}^{l}$ are just about the lengths of the intervals $\tilde{a}_{\alpha}$ and $\tilde{a}_{\beta}$, respectively. Thus, $V_{\mu}(\tilde{a})$ and $V_{v}(\tilde{a})$ may be respectively regarded as the global spreads of the membership function $\mu_{\tilde{a}}(x)$ and non-membership function $v_{\tilde{a}}(x)$. Clearly, $V_{\mu}(\tilde{a})$ and $V_{v}(\tilde{a})$ basically measure how much there is uncertainty and vagueness in the TIFN $\tilde{a}=\left((\underline{a}, a, \bar{a}) ; w_{\tilde{a}}, u_{\tilde{a}}\right)$.

Definition 12. For a TIFN $\tilde{a}=\left((\underline{a}, a, \bar{a}) ; w_{\tilde{a}}, u_{\tilde{a}}\right)$, the possibility standard deviation of membership function is defined as follows:

$$
D_{\mu}(\tilde{a})=\sqrt{V_{\mu}(\tilde{a})}=\sqrt{\frac{1}{24}} w_{\tilde{a}}(\bar{a}-\underline{a}),
$$

the possibility standard deviation of non-membership function is defined as follows:

$$
D_{v}(\tilde{a})=\sqrt{V_{v}(\tilde{a})}=\sqrt{\frac{1}{24}\left(3+u_{\tilde{a}}\right)\left(1-u_{\tilde{a}}\right)}(\bar{a}-\underline{a}) .
$$

\subsection{A new lexicographic ranking method of TIFNs considering the risk attitude of DM}

As the stated earlier, the possibility mean $m_{\mu}(\tilde{a})$ and $m_{v}(\tilde{a})$ may be respectively regarded as a central value of the membership function $\mu_{\tilde{a}}(x)$ and nonmembership function $v_{\tilde{a}}(x)$. The possibility variance $V_{\mu}(\tilde{a})$ and $V_{v}(\tilde{a})$ may be respectively regarded as the global spreads of the membership function $\mu_{\tilde{a}}(x)$ and non-membership function $v_{\tilde{a}}(x)$. Therefore, from the membership function $\mu_{\tilde{a}}(x)$ point of view, the larger the possibility mean $m_{\mu}(\tilde{a})$, the bigger the TIFN $\tilde{a}=\left((\underline{a}, a, \bar{a}) ; w_{\tilde{a}}, u_{\tilde{a}}\right)$; the smaller the possibility variance $V_{\mu}(\tilde{a})$, the bigger the TIFN $\tilde{a}=\left((\underline{a}, a, \bar{a}) ; w_{\tilde{a}}, u_{\tilde{a}}\right)$. Likely, from the non-membership function $v_{\tilde{a}}(x)$ point of view, the larger the possibility mean $m_{v}(\tilde{a})$, the bigger the TIFN $\tilde{a}=\left((\underline{a}, a, \bar{a}) ; w_{\tilde{a}}, u_{\tilde{a}}\right)$; the smaller the possibility variance $V_{v}(\tilde{a})$, the bigger the $\operatorname{TIFN} \tilde{a}=\left((\underline{a}, a, \bar{a}) ; w_{\tilde{a}}, u_{\tilde{a}}\right)$.

Let $m_{\mu}\left(\tilde{a}_{i}\right)$ and $m_{v}\left(\tilde{a}_{i}\right)$ be the possibility means of membership and non-membership functions for TIFNs $\tilde{a}_{i}(i=1,2)$. Let $D_{\mu}\left(\tilde{a}_{i}\right)$ and $D_{v}\left(\tilde{a}_{i}\right)$ be the possibility standard deviations of membership and nonmembership functions for TIFNs $\tilde{a}_{i}(i=1,2)$. The rank indices of membership and non-membership functions for TIFN $\tilde{a}_{i}$ are respectively defined as follows:

and

$$
R_{\mu}\left(\tilde{a}_{i}, \lambda\right)=m_{\mu}\left(\tilde{a}_{i}\right)-\lambda D_{\mu}\left(\tilde{a}_{i}\right)
$$

$$
R_{v}\left(\tilde{a}_{i}, \lambda\right)=m_{v}\left(\tilde{a}_{i}\right)-\lambda D_{v}\left(\tilde{a}_{i}\right),
$$

where the parameter $\lambda$ represents the risk attitude of DM. $\lambda \in[0,0.5)$ shows that the DM is risk-like; $\lambda=0.5$ shows that the DM is risk-neutral; $\lambda \in(0.5,1]$ shows that the DM is risk-averse.

Hereby, considering the risk attitude of DM, we develop a new lexicographic ranking method for TIFNs, which can be summarized as follows:

If $R_{\mu}\left(\tilde{a}_{1}, \lambda\right)<R_{\mu}\left(\tilde{a}_{2}, \lambda\right)$, then $\tilde{a}_{1}$ is smaller than $\tilde{a}_{2}$, denoted by $\tilde{a}_{1}<\tilde{a}_{2}$;

If $R_{\mu}\left(\tilde{a}_{1}, \lambda\right)>R_{\mu}\left(\tilde{a}_{2}, \lambda\right)$, then $\tilde{a}_{1}$ is bigger than $\tilde{a}_{2}$, denoted by $\tilde{a}_{1}>\tilde{a}_{2}$;

If $R_{\mu}\left(\tilde{a}_{1}, \lambda\right)=R_{\mu}\left(\tilde{a}_{2}, \lambda\right)$, then

(i) If $R_{v}\left(\tilde{a}_{1}, \lambda\right)<R_{v}\left(\tilde{a}_{2}, \lambda\right)$, then $\tilde{a}_{1}<\tilde{a}_{2}$;

(ii) If $R_{v}\left(\tilde{a}_{1}, \lambda\right)=R_{v}\left(\tilde{a}_{2}, \lambda\right)$, then $\tilde{a}_{1}$ and $\tilde{a}_{2}$ represent the same information, denoted by $\tilde{a}_{1}=\tilde{a}_{2}$;

(iii) If $R_{v}\left(\tilde{a}_{1}, \lambda\right)>R_{v}\left(\tilde{a}_{2}, \lambda\right)$, then $\tilde{a}_{1}>\tilde{a}_{2}$.

Remark 1. It is easily seen that $R_{\mu}\left(\tilde{a}_{i}, \lambda\right)$ and $R_{v}\left(\tilde{a}_{i}, \lambda\right)$ are the linear functions of a TIFN $\tilde{a}_{i}$, i.e., $R_{\mu}\left(\tilde{a}_{1}+\tilde{a}_{2}, \lambda\right)=R_{\mu}\left(\tilde{a}_{1}, \lambda\right)+R_{\mu}\left(\tilde{a}_{2}, \lambda\right) \quad$ and $R_{v}\left(\tilde{a}_{1}+\tilde{a}_{2}, \lambda\right)=R_{v}\left(\tilde{a}_{1}, \lambda\right)+R_{v}\left(\tilde{a}_{2}, \lambda\right) \quad$ with $\quad w_{\tilde{a}_{1}}=w_{\tilde{a}_{2}}$ and $u_{\tilde{a}_{1}}=u_{\tilde{a}_{2}}$. However, the ratio $R(\tilde{a}, \lambda)$ of the value index to the ambiguity index defined in $\mathrm{Li}^{7}$ is not a linear function of a TIFN $\tilde{a}$.

Remark 2. The notable advantage of the above ranking method is that it can sufficiently consider the risk preference of DM and is thus more reasonable in practical application (see Subsection 5.1 in detail).

\section{MAGDM with TIFNs and incomplete weight information}


In this section, we adopt the above ranking method of TIFNs to solve MAGDM problems in which the ratings of alternatives on attributes are expressed using TIFNs and the attribute weight information is incomplete. The corresponding group decision method is then proposed.

\subsection{Presentation for MAGDM using TIFNs with incomplete weight information}

A MAGDM problem is to find a best compromise solution from all feasible alternatives assessed on multiple attributes. Assume that there is a group consisting of $k$ DMs $\left\{P_{1}, P_{2}, \cdots, P_{k}\right\}$ who have to choose one of (or rank) $m$ alternatives $\left\{A_{1}, A_{2}, \cdots, A_{m}\right\}$ based on $n$ attributes $\left\{a_{1}, a_{2}, \cdots, a_{n}\right\}$. Denote an alternative set by $A=\left\{A_{1}, A_{2}, \cdots, A_{m}\right\} \quad$ and an attribute set by $F=\left\{a_{1}, a_{2}, \cdots, a_{n}\right\}$. The weight vector of DMs is $\boldsymbol{v}=\left(v_{1}, v_{2}, \cdots, v_{k}\right)^{\mathrm{T}}$, satisfying that $0 \leq v_{t} \leq 1$ and $\sum_{t=1}^{k} v_{t}=1$. Suppose that the intuitionistic fuzzy rating of an alternative $A_{i}$ on an attribute $a_{j}$ given by the DM $P_{t}$ is a TIFN $\tilde{a}_{i j}^{(t)}=\left(\left(\underline{a}_{i j}^{(t)}, a_{i j}^{(t)}, \bar{a}_{i j}^{(t)}\right) ; w_{i j}^{(t)}, u_{i j}^{(t)}\right)$, where $w_{i j}^{(t)}$ and $u_{i j}^{(t)}$ represent the maximum degree of membership and the minimum degree of non-membership, respectively, such that they satisfy the conditions: $0 \leq w_{i j}^{(t)} \leq 1,0 \leq u_{i j}^{(t)} \leq 1$, and $w_{i j}^{(t)}+u_{i j}^{(t)} \leq 1$. Hence, a MAGDM problem using TIFNs can be concisely expressed in matrix format as follows:

$$
\tilde{A}^{(t)}=\left(\tilde{a}_{i j}^{(t)}\right)_{m \times n}(t=1,2, \cdots, k),
$$

which are the triangular intuitionistic fuzzy decision matrixes.

In some real decision situations, the importance for different attributes should be considered. Let $\boldsymbol{\omega}=\left(\omega_{1}, \omega_{2}, \cdots, \omega_{n}\right)^{\mathrm{T}}$ be the weight vector of attributes $a_{j}(j=1,2, \cdots, n)$, satisfying that $\omega_{j} \geq 0(j=1,2, \cdots, n)$ and $\sum_{j=1}^{n} \omega_{j}=1$. A set of all weight vectors is denoted by $\Lambda_{0}$,

i.e., $\Lambda_{0}=\left\{\boldsymbol{\omega}=\left(\omega_{1}, \omega_{2}, \cdots, \omega_{n}\right)^{\mathrm{T}} \mid \sum_{j=1}^{n} \omega_{j}=1, \omega_{j} \geq 0, j=1,2, \cdots, n\right\}$. In many conditions, some information on attribute weights may be acquired. These weight preference information may be described using different forms such as constraint relations or order relations. Usually incomplete information of attribute weights can be obtained according to partial preference relations on weights given by the DM and has several different structure forms. Summarizing earlier research, $\mathrm{Li}^{24}$ mathematically and rigorously expressed these weight information structures in the following five basic relations among attribute weights, which are denoted by subsets $\Lambda_{s} \quad(s=1,2,3,4,5)$ of weight vectors in $\Lambda_{0}$, respectively (Wan and $\mathrm{Li}^{25} ; \mathrm{Li}$ and $\mathrm{Wan}^{26}$ ).

(1) The set of weights expressing a weak ranking: $\Lambda_{1}=\left\{\boldsymbol{\omega} \in \Lambda_{0} \mid \omega_{t} \geq \omega_{j}\right.$ for all $t \in T_{1}$ and $\left.j \in J_{1}\right\}$, where $T_{1}$ and $J_{1}$ are two disjoint subsets of the subscript index set $N=\{1,2, \cdots, n\}$ of all attributes. Thus, $\Lambda_{1}$ is a set of all weight vectors in $\Lambda_{0}$ with the property that the weight of an attribute in the set $T_{1}$ is greater than or equal to that of an attribute in the set $J_{1}$.

(2) The set of weights expressing a strict ranking: $\Lambda_{2}=\left\{\boldsymbol{\omega} \in \Lambda_{0} \mid \beta_{t j} \geq \omega_{t}-\omega_{j} \geq \alpha_{t j}\right.$ for all $t \in T_{2}$ and $\left.j \in J_{2}\right\}$ , where $\alpha_{t j}>0$ and $\beta_{t j}>0$ are constants, satisfying $\beta_{t j}>\alpha_{t j} ; T_{2}$ and $J_{2}$ are two disjoint subsets of $N$. Thus, $\Lambda_{2}$ is a set of all weight vectors in $\Lambda_{0}$ with the property that the weight of an attribute in the set $T_{2}$ is greater than or equal to that of an attribute in the set $J_{2}$ but their difference does not exceed some range, i.e., a closed interval $\left[\alpha_{t j}, \beta_{t j}\right]$.

(3) The set of weights expressing a ranking with multiples: $\Lambda_{3}=\left\{\boldsymbol{\omega} \in \Lambda_{0} \mid \omega_{t} \geq \xi_{t j} \omega_{j}\right.$ for all $t \in T_{3}$ and $\left.j \in J_{3}\right\}$, where $\xi_{t j}>0$ is a constant; $T_{3}$ and $J_{3}$ are two disjoint subsets of $M$. Thus, $\Lambda_{3}$ is a set of all weight vectors in $\Lambda_{0}$ with the property that the weight of an attribute in the set $T_{3}$ is greater than or equal to $\xi_{t j}$ multiple of that of an attribute in the set $J_{3}$.

(4) The set of weights expressing an interval form: $\Lambda_{4}=\left\{\omega \in \Lambda_{0} \mid \gamma_{j} \geq \omega_{j} \geq \eta_{j}\right.$ for all $\left.j \in J_{4}\right\} \quad, \quad$ where $\gamma_{j}>0$ and $\eta_{j}>0$ are constants, satisfying $\gamma_{j}>\eta_{j} ; J_{4}$ is a subset of $N$. Thus, $\Lambda_{4}$ is a set of all weight vectors in $\Lambda_{0}$ with the property that the weight of an attribute in the set $J_{4}$ does not exceed some range, i.e., a closed interval $\left[\eta_{j}, \gamma_{j}\right]$.

(5) The set of weights expressing a ranking of differences: $\quad \Lambda_{5}=\left\{\boldsymbol{\omega} \in \Lambda_{0} \mid \omega_{t}-\omega_{j} \geq \omega_{k}-\omega_{s}\right.$ for all $t \in T_{5}, j \in J_{5}, k \in K_{5}$ and $\left.l \in L_{5}\right\}$, where $T_{5}, J_{5}, K_{5}$ and $L_{5}$ are four disjoint subsets of $N$. Thus, $\Lambda_{5}$ is a set of all weight vectors in $\Lambda_{0}$ with the property that the difference between weights of attributes in the sets $T_{5}$ and $J_{5}$, is greater than or equal to that of attributes in the sets $K_{5}$ and $L_{5}$.

Cases (1)-(4) are well known types of imprecise information, and Case (5) is a ranking of differences of adjacent parameters obtained by weak rankings among 
the parameters, which can be subsequently constructed based on Case (1).

In reality, usually the preference information structure $\Lambda$ of attribute importance may consist of several sets of the above basic sets $\Lambda_{s}(s=1,2,3,4,5)$. For example, the DM may provide a preference information structure expressed as follows $\left(\mathrm{Li}^{24}\right)$ :

$$
\begin{gathered}
\hat{\Lambda}=\left\{\boldsymbol{\omega} \in \Lambda_{0} \mid 0.15 \leq \omega_{1} \leq 0.55,0.2 \leq \omega_{2} \leq 0.65,\right. \\
\left.0.1 \leq \omega_{3} \leq 0.35, \omega_{2} \geq 1.2 \omega_{1}, 0.02 \leq \omega_{2}-\omega_{3} \leq 0.45\right\},
\end{gathered}
$$

which may be decomposed into the following three basic subsets: $\hat{\Lambda}_{2}=\left\{\omega \in \Lambda_{0} \mid 0.02 \leq \omega_{2}-\omega_{3} \leq 0.45\right\}$, $\hat{\Lambda}_{3}=\left\{\boldsymbol{\omega} \in \Lambda_{0} \mid \omega_{2} \geq 1.2 \omega_{1}\right\} \quad$ and $\quad \hat{\Lambda}_{4}=\left\{\boldsymbol{\omega} \in \hat{\Lambda}_{0} \mid 0.15 \leq \omega_{1} \leq 0.55\right.$, $\left.0.2 \leq \omega_{2} \leq 0.65,0.1 \leq \omega_{3} \leq 0.35\right\}$ where $\Lambda_{0}=\left\{\boldsymbol{\omega}=\left(\omega_{j}\right)_{3 \times 1} \mid \omega_{1}+\omega_{2}+\omega_{3}=1, \omega_{j} \in[0,1](j=1,2,3)\right\}$. In other words, the information structure $\hat{\Lambda}$ consists of the above three sets $\hat{\Lambda}_{2}, \hat{\Lambda}_{3}$ and $\hat{\Lambda}_{4}$.

The MAGDM problem considered in this paper is to determine the best alternative from the finite alternative set according to the matrixes $\tilde{\boldsymbol{A}}^{(t)}=\left(\tilde{a}_{i j}^{(t)}\right)_{m \times n}$ $(t=1,2, \cdots, k)$, and the weight information $\Lambda$.

\subsection{A new method for MAGDM with TIFNs}

In general, attributes can be classified into two types: benefit attributes and cost attributes. In other words, the attribute set $F$ can be divided into two subsets: $F_{1}$ and $F_{2}$, which are the subsets of benefit attributes and cost attributes, respectively. Since the $n$ attributes may be measured in different ways, the matrix $\tilde{\boldsymbol{A}}^{(t)}=\left(\tilde{a}_{i j}^{(t)}\right)_{m \times n}$ needs to be normalized into $\tilde{\boldsymbol{R}}^{(t)}=\left(\tilde{r}_{i j}^{(t)}\right)_{m \times n}$, where $\tilde{r}_{i j}^{(t)}=\left(\left(\underline{r i j}_{i j}^{(t)}, r_{i j}^{(t)}, \bar{r}_{i j}^{(t)}\right) ; w_{i j}^{(t)}, u_{i j}^{(t)}\right)$. In this paper, the normalization method is chosen as follows (Li et al. ${ }^{8}$ ):

$$
\begin{aligned}
& \left(r_{i j}^{(t)}, r_{i j}^{(t)}, \bar{r}_{i j}^{(t)}\right)=\left(\frac{a_{i j}^{(t)}}{\bar{a}_{i j}^{(t)+}}, \frac{a_{i j}^{(t)}}{\bar{a}_{i j}^{(t)+}}, \frac{\bar{a}_{i j}^{(t)}}{\bar{a}_{i j}^{(t)+}}\right) \text { for } j \in F_{1} ; \\
& \left(\underline{r}_{i j}^{(t)}, r_{i j}^{(t)}, \bar{r}_{i j}^{(t)}\right)=\left(\frac{a_{i j}^{(t)-}}{\bar{a}_{i j}^{(t)}}, \frac{a_{i j}^{(t)-}}{a_{i j}^{(t)}}, \frac{a_{i j}^{(t)-}}{\underline{a}_{i j}^{(t)}}\right) \text { for } j \in F_{2},
\end{aligned}
$$

where $\quad \bar{a}_{i j}^{(t)+}=\max \left\{\bar{a}_{i j}^{(t)} \mid i=1,2, \cdots, m\right\} \quad, \quad$ and $\underline{a}_{i j}^{(t)-}=\min \left\{\underline{a}_{i j}^{(t)} \mid i=1,2, \cdots, m\right\}$.

Used the weighted average operator of TIFNs $\varphi_{\omega}$ (i.e., Eq. (2)) to integrate all the attribute values of alternative $A_{i}$ given by the $\mathrm{DM} P_{t}$, the individual overall attribute value of alternative $A_{i}$ given by $P_{t}$ is obtained as follows:

$$
\tilde{a}_{i}^{(t)}=\varphi_{\omega}\left(\tilde{r}_{i 1}^{(t)}, \tilde{r}_{i 2}^{(t)}, \cdots, \tilde{r}_{i n}^{(t)}\right)=\left(\left(\sum_{j=1}^{n} \omega_{j} r_{i j}^{(t)}, \sum_{j=1}^{n} \omega_{j} r_{i j}^{(t)}, \sum_{j=1}^{n} \omega_{j} \bar{r}_{i j}^{(t)}\right) ;\right.
$$

$$
\left.\widehat{1 \leq j \leq n} w_{i j}^{(t)}, \underset{1 \leq j \leq n}{\vee} u_{i j}^{(t)}\right),
$$

where $\boldsymbol{\omega}=\left(\omega_{1}, \omega_{2}, \cdots, \omega_{n}\right)^{\mathrm{T}}$ is the weight vector of attributes.

Used the weighted average operator of TIFNs $\varphi_{v}$ (i.e., Eq. (2)) to integrate all $\tilde{a}_{i}^{(t)} \quad(t=1,2, \cdots, k)$, the collective overall attribute value of alternative $A_{i}$ is obtained as follows:

$$
\begin{gathered}
\tilde{a}_{i}=\varphi_{v}\left(\tilde{a}_{i}^{(1)}, \tilde{a}_{i}^{(2)}, \cdots, \tilde{a}_{i}^{(k)}\right)=\left(\left(\sum_{t=1}^{k} \sum_{j=1}^{n} v_{t} \omega_{j} r_{j j}^{(t)}, \sum_{t=1}^{k} \sum_{j=1}^{n} v_{t} \omega_{j} r_{i j}^{(t)},\right.\right. \\
\left.\sum_{t=1}^{k} \sum_{j=1}^{n} v_{t} \omega_{j} \bar{r}_{i j}^{(t)}\right) ; \widehat{1}_{1 \leq t \leq k, 1 \leq j \leq n} w_{i j}^{(t)}, \underbrace{\vee}_{1 \leq t \leq k, 1 \leq j \leq n} u_{i j}^{(t)}),
\end{gathered}
$$

where $\boldsymbol{v}=\left(v_{1}, v_{2}, \cdots, v_{k}\right)^{\mathrm{T}}$ is the weight vector of the DMs, symbols $\bigwedge_{1 \leq t \leq k, 1 \leq j \leq n}$ and $\underset{1 \leq t \leq k, 1 \leq j \leq n}{\vee}$ respectively represent the max and min operators about variables $t$ and $j$, which are denoted by $\underset{t, j}{\wedge}$ and $\underset{t, j}{\vee}$ for simplicity.

By Definitions 9 and 12, and Eqs. (25) and (26), the possibility means, standard deviations and ranking indices of membership and non-membership functions for the collective overall attribute value $\tilde{a}_{i}$ of alternative $A_{i}$ are computed as follows:

$$
\begin{gathered}
m_{\mu}\left(\tilde{a}_{i}\right)=\frac{1}{6}\left(\bigwedge_{t, j} w_{i j}^{(t)}\right)^{2} \sum_{t=1}^{k} \sum_{j=1}^{n}\left[v_{t} \omega_{j}\left(\underline{r}_{i j}^{(t)}+r_{i j}^{(t)}+\bar{r}_{i j}^{(t)}\right)\right], \\
m_{v}\left(\tilde{a}_{i}\right)=\frac{1}{6}\left(1-\bigvee_{t, j} u_{i j}^{(t)}\right)\left[2\left(1+2 \bigvee_{t, j} u_{i j}^{(t)}\right) \sum_{t=1}^{k} \sum_{j=1}^{n} v_{t} \omega_{j} r_{i j}^{(t)}\right. \\
\left.+\left(2+\bigvee_{t, j}^{u_{i j}^{(t)}}\right) \sum_{t=1}^{k} \sum_{j=1}^{n} v_{t} \omega_{j}\left(\underline{r}_{i j}^{(t)}+\bar{r}_{i j}^{(t)}\right)\right] . \\
D_{\mu}\left(\tilde{a}_{i}\right)=\sqrt{\frac{1}{24}}\left(\bigwedge_{t, j}^{w_{i j}^{(t)}}\right) \sum_{t=1}^{k} \sum_{j=1}^{n}\left[v_{t} \omega_{j}\left(\bar{r}_{i j}^{(t)}-\underline{r}_{i j}^{(t)}\right)\right], \\
D_{v}\left(\tilde{a}_{i}\right)=\sqrt{\frac{1}{24}\left(3+\bigvee_{t, j}^{u_{i j}^{(t)}}\right)\left(1-\bigvee_{t, j}^{u_{i j}^{(t)}}\right) \sum_{t=1}^{k} \sum_{j=1}^{n} v_{t} \omega_{j}\left(\bar{r}_{i j}^{(t)}-r_{i j}^{(t)}\right),} \\
R_{\mu}\left(\tilde{a}_{i}, \lambda\right)=\frac{1}{6}\left(\bigwedge_{t, j}^{w_{i j}^{(t)}}\right)^{2} \sum_{t=1}^{k} \sum_{j=1}^{n}\left[v_{t} \omega_{j}\left(r_{i j}^{(t)}+r_{i j}^{(t)}+\bar{r}_{i j}^{(t)}\right)\right] \\
-\lambda \sqrt{\frac{1}{24}}\left(\bigwedge_{t, j}^{w_{i j}^{(t)}}\right) \sum_{t=1}^{k} \sum_{j=1}^{n}\left[v_{t} \omega_{j}\left(\bar{r}_{i j}^{(t)}-\underline{r}_{i j}^{(t)}\right)\right]
\end{gathered}
$$

and

$$
\begin{gathered}
R_{v}\left(\tilde{a}_{i}, \lambda\right)=\frac{1}{6}\left(1-\bigvee_{t, j} u_{i j}^{(t)}\right)\left[2\left(1+2 \bigvee_{t, j} u_{i j}^{(t)}\right) \sum_{t=1}^{k} \sum_{j=1}^{n} v_{t} \omega_{j} r_{i j}^{(t)}\right. \\
\left.+\left(2+\bigvee_{t, j} u_{i j}^{(t)}\right) \sum_{t=1}^{k} \sum_{j=1}^{n} v_{t} \omega_{j}\left(r_{i j}^{(t)}+\bar{r}_{i j}^{(t)}\right)\right]
\end{gathered}
$$


$-\lambda \sqrt{\frac{1}{24}\left(3+\underset{t, j}{\mathcal{M}_{i j}^{(t)}}\right)\left(1-\bigvee_{t, j} u_{i j}^{(t)}\right)} \sum_{t=1}^{k} \sum_{j=1}^{n} v_{t} \omega_{j}\left(\bar{r}_{i j}^{(t)}-\underline{r}_{i j}^{(t)}\right)$,

respectively.

It is easy to see that the ranking indices $R_{\mu}\left(\tilde{a}_{i}, \lambda\right)$ and $R_{v}\left(\tilde{a}_{i}, \lambda\right)$ are the linear functions of the attribute weight vector $\boldsymbol{\omega}=\left(\omega_{1}, \omega_{2}, \cdots, \omega_{n}\right)^{\mathrm{T}}$, simply denoted by $z_{1 i}(\omega)=R_{\mu}\left(\tilde{a}_{i}, \lambda\right)$ and $z_{2 i}(\omega)=R_{v}\left(\tilde{a}_{i}, \lambda\right)$, respectively.

According to the ranking method of TIFNs in subsection 3.3, the bigger the ranking indices of membership and non-membership functions for the collective overall attribute value, the better the alternative. Therefore, the reasonable weight vector of attributes $\omega=\left(\omega_{1}, \omega_{2}, \cdots, \omega_{n}\right)^{\mathrm{T}}$ should be obtained so that all the ranking indices of membership and nonmembership functions for alternatives could be as big as possible. Thereby, the following multi-objective mathematical programming model is set up:

$$
\begin{aligned}
& \max z_{1 i}(\boldsymbol{\omega})=R_{\mu}\left(\tilde{a}_{i}, \lambda\right)(i=1,2, \cdots, m) \\
& \max z_{2 i}(\boldsymbol{\omega})=R_{v}\left(\tilde{a}_{i}, \lambda\right)(i=1,2, \cdots, m) \\
& \text { s.t. } \boldsymbol{\omega} \in \Lambda
\end{aligned}
$$

Since there is no preference between the alternatives, Eq. (37) can be transformed into the biobjective mathematical programming models using the equal weight sum method as follows:

$$
\begin{aligned}
& \max \left\{Z_{1}(\boldsymbol{\omega}), Z_{2}(\boldsymbol{\omega})\right\}, \\
& \text { s.t. } \boldsymbol{\omega} \in \Lambda
\end{aligned}
$$

where $Z_{1}(\omega)$ and $Z_{2}(\omega)$ are respectively the ranking indices of membership and non-membership functions for all alternatives, defined as follows:

$$
\begin{aligned}
& Z_{1}(\boldsymbol{\omega})=\sum_{i=1}^{m} z_{1 i}(\boldsymbol{\omega})=\sum_{i=1}^{m} R_{\mu}\left(\tilde{a}_{i}, \lambda\right), \\
& Z_{2}(\boldsymbol{\omega})=\sum_{i=1}^{m} z_{2 i}(\boldsymbol{\omega})=\sum_{i=1}^{m} R_{v}\left(\tilde{a}_{i}, \lambda\right) .
\end{aligned}
$$

Obviously, Eq. (38) is a bi-objective linear programming model on the decision variable vector $\boldsymbol{\omega}=\left(\omega_{1}, \omega_{2}, \cdots, \omega_{n}\right)^{\mathrm{T}}$. There are few standard ways of defining a solution of multi-objective programming. However, in this study we focus on developing a membership function based weighted sum approach to solving Eq. (38).

In order to do that, we first solve the following four linear programming models:

$$
\begin{aligned}
& \max Z_{1}(\omega), \\
& \text { s.t. } \omega \in \Lambda, \\
& \min Z_{1}(\omega), \\
& \text { s.t. } \omega \in \Lambda \\
& \max Z_{2}(\omega) \\
& \text { s.t. } \omega \in \Lambda
\end{aligned}
$$

and

$$
\begin{aligned}
& \min Z_{2}(\omega) \\
& \text { s.t. } \omega \in \Lambda
\end{aligned}
$$

Let $Z_{1}^{\max }, Z_{1}^{\min }, Z_{2}^{\max }$ and $Z_{2}^{\min }$ be the optimal objective values of Eqs. (41)-(44), respectively. Then, the membership functions for the two objective functions of Eq. (38) can be defined as follows:

$$
\mu_{Z_{1}}(\boldsymbol{\omega})=\frac{Z_{1}(\boldsymbol{\omega})-Z_{1}^{\min }}{Z_{1}^{\max }-Z_{1}^{\min }}, \mu_{Z_{2}}(\boldsymbol{\omega})=\frac{Z_{2}(\boldsymbol{\omega})-Z_{2}^{\min }}{Z_{2}^{\max }-Z_{2}^{\min }} .
$$

Thus, Eq. (38) can be transformed into the following linear programming model:

$$
\begin{aligned}
& \max Z(\boldsymbol{\omega})=\theta_{1} \mu_{Z_{1}}(\boldsymbol{\omega})+\theta_{2} \mu_{Z_{2}}(\boldsymbol{\omega}), \\
& \text { s.t. } \boldsymbol{\omega} \in \Lambda
\end{aligned}
$$

where $\theta_{1}$ and $\theta_{2}$ are respectively the objective weights or importance of $\mu_{Z_{1}}(\omega)$ and $\mu_{Z_{2}}(\omega)$, and satisfy that $0 \leq \theta_{1} \leq 1,0 \leq \theta_{2} \leq 1$ and $\theta_{1}+\theta_{2}=1 . \theta_{1}>\theta_{2}$ shows that the DM prefers to certainty or positive feeling; $\theta_{1}<\theta_{2}$ shows that the DM prefers to uncertainty or negative feeling; $\theta_{1}=\theta_{2}=0.5$ shows that the DMs is indifferent between positive or negative feeling.

By using the Simplex Method to solve Eq. (46), the optimal attribute weight vector $\omega^{*}$ can be obtained. Thus, the ranking indices $R_{\mu}\left(\tilde{a}_{i}, \lambda\right)$ and $R_{v}\left(\tilde{a}_{i}, \lambda\right)$ are calculated by Eqs. (35) and (36).

In sum, an algorithm and process of the MAGDM problems with TIFNs may be given as follows:

Step 1 The DMs identifies the valuation attributes;

Step 2 Construct the fuzzy decision matrix $\tilde{\boldsymbol{A}}^{(t)}=\left(\tilde{a}_{i j}^{(t)}\right)_{m \times n} ;$

Step 3 Normalize the decision matrix $\tilde{\boldsymbol{A}}^{(t)}=\left(\tilde{a}_{i j}^{(t)}\right)_{m \times n}$ to $\tilde{\boldsymbol{R}}^{(t)}=\left(\tilde{r}_{i j}^{(t)}\right)_{m \times n}$ according to Eqs. (27) and (28);

Step 4 Compute the collective overall attribute value of alternative $A_{i}$ by Eqs. (29) and (30);

Step 5 Construct Eq. (46) through solving Eqs. (41)-(44);

Step 6 Choose the risk attitude parameter value $\lambda$ and solve Eq. (46) to obtain the optimal attribute weight vector of $\omega^{*}$;

Step 7 Calculate the ranking indices $\left.R_{\mu}\left(\tilde{a}_{i}, \lambda\right)\right)$ and $R_{v}\left(\tilde{a}_{i}, \lambda\right)$ ) of membership and non-membership functions for the collective overall attribute value of alternative $A_{i}$ by Eqs. (35) and (36);

Step 8 According to the ranking indices $R_{\mu}\left(\tilde{a}_{i}, \lambda\right)$ ) and $\left.R_{v}\left(\tilde{a}_{i}, \lambda\right)\right)$ of alternative $A_{i} \quad(i=1,2, \cdots, m)$, the ranking order of alternatives is generated by the ranking 
method based on possibility mean and standard deviation in subsection 3.3.

\section{A personnel selection problem and comparison analysis of computational results}

In this section, a personnel selection problem is illustrated to demonstrate the applicability and implementation process of the MAGDM method proposed in this paper. The comparison analysis of computational results is also conducted to show the superiority of the proposed method.

\subsection{A personnel selection problem and the analysis process}

The proposed method is illustrated with a personnel selection problem adapted from $\mathrm{Li}^{7}$. Suppose that a software company desires to hire a system analyst. After preliminary screening, three candidates (i.e., alternatives) $A_{1}, A_{2}$ and $A_{3}$ remain for further evaluation. The decision making committee consists of three DMs $P_{1}, P_{2}$ and $P_{3}$. They assesses the three candidates on the basis of five attributes, including emotional steadiness $a_{1}$, oral communication skills $a_{2}$, personality $a_{3}$, past experience $a_{4}$ and self-confidence $a_{5}$. The weight vector of DMs is $v=(0.40,0.25,0.35)^{\mathrm{T}}$. After the data acquisition and statistical treatment, the ratings of the candidates with respect to attributes can be represented by TIFNs as in Tables 1-3, where $((5.7,7.7,9.3) ; 0.7,0.2)$ in the Table 1 is an TIFN which indicates that the mark of the candidate $A_{1}$ with respect to the attribute $a_{1}$ is about 7.7 with the maximum satisfaction degree is 0.7 , while the minimum dissatisfaction degree is 0.2 . In other words, the hesitation degree is 0.1 . Other TIFNs in Tables 1-3 are explained similarly.

With ever increasing complexity in real personnel selection management, it is very difficult for the DMs to give precisely the five weights a priori. Sometimes, the DMs may pay more attention to importance of some attributes, i.e., specify some preference relations on weights of attributes according to their knowledge, experience and judgment. For example, the pair-wise comparison may be more practical for weighting attributes. Thus, some preference information is acquired. In this example, the known weight preference information provided by the DMs is as follows:

$$
\begin{gathered}
\Lambda=\left\{\omega_{2} \geq 0.13 \omega_{1}, 0.05 \leq \omega_{1}-\omega_{3} \leq 0.28,\right. \\
\left.0.11 \leq \omega_{3} \leq 0.29, \omega_{4}-\omega_{2} \geq \omega_{1}-\omega_{5}, \omega_{5} \geq \omega_{4}\right\}
\end{gathered}
$$

Table 1 The TIFN decision matrix given by $P_{1}$

\begin{tabular}{ccc}
\hline \multicolumn{1}{c}{$A_{1}$} & \multicolumn{1}{c}{$A_{2}$} & $A_{3}$ \\
\hline$a_{1}((5.7,7.7,9.3) ; 0.7,0.2)$ & $((6.5,8.6,10) ; 0.4,0.5)$ & $((6.5,8.2,9.3) ; 0.8,0.1)$ \\
$a_{2}((5.0,7.0,9.0) ; 0.6,0.3)$ & $((8.0,9.0,10) ; 0.6,0.3)$ & $((7.0,9.0,10) ; 0.7,0.2)$ \\
$a_{3}((5.7,7.7,9.0) ; 0.8,0.1)$ & $((8.3,9.7,10) ; 0.7,0.2)$ & $((7.0,9.0,10) ; 0.5,0.2)$ \\
$a_{4}((8.33,9.67,10) ; 0.6,0.4)$ & $((8.0,9.0,10) ; 0.6,0.3)$ & $((6.0,8.0,9.0) ; 0.6,0.2)$ \\
$a_{5}((3.0,5.0,7.0) ; 0.6,0.3)$ & $((7.0,9.0,10) ; 0.6,0.2)$ & $((6.3,8.3,9.7) ; 0.7,0.2)$ \\
\hline
\end{tabular}

Table 2 The TIFN decision matrix given by $P_{2}$

\begin{tabular}{ccc}
\hline$A_{1}$ & $A_{2}$ & $A_{3}$ \\
\hline$a_{1}((3.2,5.2,8.6) ; 0.4,0.5)$ & $((5.8,8.4,9.4) ; 0.3,0.6)$ & $((4.5,7.2,10) ; 0.7,0.2)$ \\
$a_{2}((2.4,8.0,10) ; 0.8,0.1)$ & $((8.1,9.1,9.7) ; 0.7,0.2)$ & $((6.7,8.9,9.7) ; 0.6,0.3)$ \\
$a_{3}((6.7,8.5,10) ; 0.6,0.3)$ & $((7.8,8.6,9.9) ; 0.6,0.3)$ & $((5.7,8.9,9.4) ; 0.3,0.6)$ \\
$a_{4}((4.8,5.6,9.1) ; 0.2,0.5)$ & $((7.8,8.1,9.6) ; 0.8,0.1)$ & $((4.6,7.8,10) ; 0.6,0.2)$ \\
$a_{5}((4.2,6.0,6.8) ; 0.5,0.3)$ & $((6.7,7.3,9.8) ; 0.5,0.3)$ & $((6.1,7.9,10) ; 0.6,0.1)$ \\
\hline
\end{tabular}


Table 3 The TIFN decision matrix given by $P_{3}$

\begin{tabular}{ccc}
\hline$A_{1}$ & $A_{2}$ & $A_{3}$ \\
\hline$a_{1}((6.7,7.2,8.7) ; 0.7,0.2)$ & $((4.5,7.6,10) ; 0.4,0.5)$ & $((7.5,8.7,9.8) ; 0.7,0.2)$ \\
$a_{2}((6.0,8.0,10) ; 0.3,0.5)$ & $((5.0,7.0,8.0) ; 0.6,0.1)$ & $((5.7,6.9,7.2) ; 0.5,0.3)$ \\
$a_{3}((6.0,7.2,10) ; 0.7,0.3)$ & $((8.5,8.7,9.3) ; 0.7,0.3)$ & $((4.7,5.9,6.4) ; 0.6,0.3)$ \\
$a_{4}((6.3,8.7,9.5) ; 0.5,0.4)$ & $((7.8,8.9,9.4) ; 0.5,0.2)$ & $((6.1,8.2,10) ; 0.7,0.2)$ \\
$a_{5}((5.0,5.8,6.9) ; 0.2,0.7)$ & $((5.7,7.9,8.7) ; 0.7,0.1)$ & $((4.3,5.3,10) ; 0.8,0.1)$ \\
\hline
\end{tabular}

Table 4 The normalized TIFN decision matrix given by $p$

\begin{tabular}{ccc}
\hline$A_{1}$ & \multicolumn{1}{c}{$A_{2}$} & \multicolumn{1}{c}{$A_{3}$} \\
\hline$a_{1}((0.57,0.77,0.93) ; 0.7,0.2)$ & $((0.65,0.86,1.0) ; 0.4,0.5)$ & $((0.65,0.82,0.93) ; 0.8,0.1)$ \\
$a_{2}((0.50,0.70,0.90) ; 0.6,0.3)$ & $((0.80,0.90,1.0) ; 0.6,0.3)$ & $((0.70,0.90,1.0) ; 0.7,0.2)$ \\
$a_{3}((0.57,0.77,0.90) ; 0.8,0.1)$ & $((0.83,0.97,1.0) ; 0.7,0.2)$ & $((0.70,0.90,1.0) ; 0.5,0.2)$ \\
$a_{4}((0.83,0.97,1.0) ; 0.6,0.4)$ & $((0.80,0.90,1.0) ; 0.6,0.3)$ & $((0.60,0.80,0.90) ; 0.6,0.2)$ \\
$a_{5}((0.30,0.50,0.70) ; 0.6,0.3)$ & $((0.70,0.90,1.0) ; 0.6,0.2)$ & $((0.63,0.83,0.97) ; 0.7,0.2)$ \\
\hline
\end{tabular}

Table 5 The normalized TIFN decision matrix given by $p_{2}$

\begin{tabular}{ccc}
\hline$A_{1}$ & $A_{2}$ & $A_{3}$ \\
\hline$a_{1}((0.32,0.52,0.86) ; 0.4,0.5)$ & $((0.58,0.84,0.94) ; 0.3,0.6)$ & $((0.45,0.72,1.0) ; 0.7,0.2)$ \\
$a_{2}((0.24,0.80,1.0) ; 0.8,0.1)$ & $((0.81,0.91,0.97) ; 0.7,0.2)$ & $((0.67,0.89,0.97) ; 0.6,0.3)$ \\
$a_{3}((0.67,0.85,1.0) ; 0.6,0.3)$ & $((0.78,0.86,0.99) ; 0.6,0.3)$ & $((0.57,0.89,0.94) ; 0.3,0.6)$ \\
$a_{4}((0.48,0.56,0.91) ; 0.2,0.5)$ & $((0.78,0.81,0.96) ; 0.8,0.1)$ & $((0.46,0.78,1.0) ; 0.6,0.2)$ \\
$a_{5}((0.42,0.60,0.68) ; 0.5,0.3)$ & $((0.67,0.73,0.98) ; 0.5,0.3)$ & $((0.61,0.79,1.0) ; 0.6,0.1)$ \\
\hline
\end{tabular}

Table 6 The normalized TIFN decision matrix given by $p_{3}$

\begin{tabular}{ccl}
\hline \multicolumn{1}{c}{$A_{1}$} & \multicolumn{1}{c}{$A_{2}$} & \multicolumn{1}{c}{$A_{3}$} \\
\hline$a_{1}((0.67,0.72,0.87) ; 0.7,0.2)$ & $((0.45,0.76,1.0) ; 0.4,0.5)$ & $((0.75,0.87,0.98) ; 0.7,0.2)$ \\
$a_{2}((0.60,0.80,1.0) ; 0.3,0.5)$ & $((0.50,0.70,0.80) ; 0.6,0.1)$ & $((0.57,0.69,0.72) ; 0.5,0.3)$ \\
$a_{3}((0.60,0.72,1.0) ; 0.7,0.3)$ & $((0.85,0.87,0.93) ; 0.7,0.3)$ & $((0.47,0.59,0.64) ; 0.6,0.3)$ \\
$a_{4}((0.63,0.87,0.95) ; 0.5,0.4)$ & $((0.78,0.89,0.94) ; 0.5,0.2)$ & $((0.61,0.82,1.0) ; 0.7,0.2)$ \\
$a_{5}((0.50,0.58,0.69) ; 0.2,0.7)$ & $(0.57,0.79,0.87) ; 0.7,0.1)$ & $((0.43,0.53,1.0) ; 0.8,0.1)$ \\
\hline
\end{tabular}

The five attributes are all benefit attributes. According to Eqs. (27) and (28), the normalized TIFN decision matrixes are obtained as in Tables 4-6.

Setting $\lambda=0.5$, we use Eqs. (31)-(36) to calculate the possibility means, standard deviations and ranking indices of membership and non-membership functions for the collective overall attribute value $\tilde{a}_{1}$ of alternative $A_{1}$ as follows:

$$
\begin{aligned}
m_{\mu}\left(\tilde{a}_{1}\right) & =0.069 \omega_{1}+0.073 \omega_{2}+0.0779 \omega_{3}+0.082 \omega_{4}+0.0548 \omega_{5}, \\
m_{v}\left(\tilde{a}_{1}\right) & =0.684 \omega_{1}+0.7187 \omega_{2}+0.773 \omega_{3}+0.8117 \omega_{4}+0.54 \omega_{5}, \\
D_{\mu}\left(\tilde{a}_{1}\right) & =0.012 \omega_{1}+0.02 \omega_{2}+0.0145 \omega_{3}+0.0117 \omega_{4}+0.012 \omega_{5}, \\
D_{v}\left(\tilde{a}_{1}\right) & =0.064 \omega_{1}+0.105 \omega_{2}+0.076 \omega_{3}+0.062 \omega_{4}+0.063 \omega_{5}, \\
z_{1}\left(\tilde{a}_{1}\right) & =R_{\mu}\left(\tilde{a}_{1}, 0.5\right) \\
& =0.063 \omega_{1}+0.063 \omega_{2}+0.07 \omega_{3}+0.076 \omega_{4}+0.049 \omega_{5}
\end{aligned}
$$

$$
\begin{aligned}
z_{2}\left(\tilde{a}_{1}\right) & =R_{v}\left(\tilde{a}_{1}, 0.5\right) \\
& =0.652 \omega_{1}+0.666 \omega_{2}+0.734 \omega_{3}+0.781 \omega_{4}+0.511 \omega_{5}
\end{aligned} .
$$

In a similar way, the ranking indices of membership and non-membership functions for other alternatives can be obtained as follows:

$$
\begin{aligned}
& z_{1}\left(\tilde{a}_{2}\right)=R_{\mu}\left(\tilde{a}_{2}, 0.5\right) \\
& =0.1054 \omega_{1}+0.1157 \omega_{2}+0.1307 \omega_{3}+0.126 \omega_{4}+0.11152 \omega_{5} \\
& z_{2}\left(\tilde{a}_{2}\right)=R_{v}\left(\tilde{a}_{2}, 0.5\right) \\
& =0.9109 \omega_{1}+0.9726 \omega_{2}+1.0869 \omega_{3}+1.0541 \omega_{4}+0.9482 \omega_{5} \\
& z_{1}\left(\tilde{a}_{3}\right)=R_{\mu}\left(\tilde{a}_{3}, 0.5\right) \\
& =0.1097 \omega_{1}+0.1107 \omega_{2}+0.1034 \omega_{3}+0.1045 \omega_{4}+0.0994 \omega_{5} \\
& z_{2}\left(\tilde{a}_{3}\right)=R_{v}\left(\tilde{a}_{3}, 0.5\right) \\
& =0.9308 \omega_{1}+0.9308 \omega_{2}+0.8730 \omega_{3}+0.9006 \omega_{4}+0.8671 \omega_{5}
\end{aligned}
$$

By Eqs. (39) and (40), we have 


$$
\begin{aligned}
& Z_{1}(\boldsymbol{\omega})=z_{1}\left(\tilde{a}_{1}\right)+z_{1}\left(\tilde{a}_{2}\right)+z_{1}\left(\tilde{a}_{3}\right) \\
& =0.2782 \omega_{1}+0.2894 \omega_{2}+0.3048 \omega_{3}+0.3068 \omega_{4}+0.2595 \omega_{5} \\
& Z_{2}(\omega)=z_{2}\left(\tilde{a}_{1}\right)+z_{2}\left(\tilde{a}_{2}\right)+z_{2}\left(\tilde{a}_{3}\right) \\
& =2.4936 \omega_{1}+2.5693 \omega_{2}+2.6942 \omega_{3}+2.7355 \omega_{4}+2.3260 \omega_{5}
\end{aligned}{ }^{\circ},
$$

According to Eq. (41), the linear programming model can be constructed as follows:

$$
\begin{aligned}
& \max Z_{1}(\boldsymbol{\omega})=\left(0.2782 \omega_{1}+0.2894 \omega_{2}+0.3048 \omega_{3}\right. \\
& \left.+0.3068 \omega_{4}+0.2595 \omega_{5}\right) \\
& \text { s.t. }\left\{\begin{array}{l}
\omega_{2} \geq 0.13 \omega_{1} \\
0.05 \leq \omega_{1}-\omega_{3} \leq 0.28 \\
0.11 \leq \omega_{3} \leq 0.29 \\
\omega_{4}-\omega_{2} \geq \omega_{1}-\omega_{5} \\
\omega_{5} \geq \omega_{4} \\
\omega_{1}+\omega_{2}+\omega_{3}+\omega_{4}=1 \\
\omega_{j} \geq 0(j=1,2,3,4,5)
\end{array}\right.
\end{aligned}
$$

By solving Eq. (47) using the Simplex method for linear programming, the optimal objective value can be obtained as $Z_{1}^{\max }=0.2877$.

Analogously, the optimal objective values can be obtained through solving Eqs. (42)-(44) as follows:

$$
Z_{1}^{\min }=0.2681, Z_{2}^{\max }=2.5649, Z_{2}^{\min }=2.3984 \text {. }
$$

Thus, according to Eq. (46) with objective weights $\theta_{1}=\theta_{2}=0.5$, the following linear programming model can be constructed:

$$
\begin{aligned}
& \max Z(\boldsymbol{\omega})=\left(14.5855 \omega_{1}+15.0986 \omega_{2}+15.8659 \omega_{3}\right. \\
& \left.+16.0412 \omega_{4}+13.6049 \omega_{5}-14.0417\right) \\
& \text { s.t. }\left\{\begin{array}{l}
\omega_{2} \geq 0.13 \omega_{1} \\
0.05 \leq \omega_{1}-\omega_{3} \leq 0.28 \\
0.11 \leq \omega_{3} \leq 0.29 \\
\omega_{4}-\omega_{2} \geq \omega_{1}-\omega_{5} \\
\omega_{5} \geq \omega_{4} \\
\omega_{1}+\omega_{2}+\omega_{3}+\omega_{4}=1 \\
\omega_{j} \geq 0(j=1,2,3,4,5)
\end{array}\right.
\end{aligned}
$$

By solving Eq. (48), the optimal weight vector of attributes is obtained as follows:

$$
\omega^{*}=(0.3221,0.0419,0.2721,0.1820,0.1820)^{\mathrm{T}} .
$$

Consequently, the ranking indices of membership and non-membership functions for the collective overall attribute values of candidates can be calculated by using Eqs. (35) and (36) as follows:

$$
\begin{aligned}
& R_{\mu}\left(\tilde{a}_{1}\right)=0.0649, R_{\mu}\left(\tilde{a}_{2}\right)=0.1176, R_{\mu}\left(\tilde{a}_{3}\right)=0.1052, \\
& R_{v}\left(\tilde{a}_{1}\right)=0.6727, R_{v}\left(\tilde{a}_{2}\right)=0.9942, R_{v}\left(\tilde{a}_{3}\right)=0.8980 .
\end{aligned}
$$

It can be easily seen that $R_{\mu}\left(\tilde{a}_{2}\right)>R_{\mu}\left(\tilde{a}_{3}\right)>R_{\mu}\left(\tilde{a}_{1}\right)$. Therefore, according to subsection 3.3, the ranking order of the candidates is $A_{2} \succ A_{3} \succ A_{1}$, the best candidate is $A_{2}$.

For any other risk attitude parameter values $\lambda \in[0,1]$ and $\theta_{1}=\theta_{2}=0.5$, in the same way, we can obtain the optimal weight vectors of attributes and the ranking indices of membership and non-membership functions for the collective overall attribute values of candidates. The computation results and ranking orders of candidates are listed in Table 7.

Table 7 The computation results for different risk attitude parameter values and ranking orders of candidates

\begin{tabular}{cccccccccc}
\hline$\lambda$ & $\omega^{*}$ & $R_{\mu}\left(\tilde{a}_{1}\right)$ & $R_{\mu}\left(\tilde{a}_{2}\right)$ & $R_{\mu}\left(\tilde{a}_{3}\right)$ & $R_{\nu}\left(\tilde{a}_{1}\right)$ & $R_{v}\left(\tilde{a}_{2}\right)$ & $R_{v}\left(\tilde{a}_{3}\right)$ & Ranking orders & Best candidates \\
\hline 0.0 & $(0.29,0.04,0.30,0.18,0.19)^{\mathrm{T}}$ & 0.0721 & 0.1175 & 0.1149 & 0.7168 & 1.0266 & 0.9421 & $A_{2} \succ A_{3} \succ A_{1}$ & $A_{2}$ \\
0.1 & $(0.29,0.04,0.30,0.18,0.19)^{\mathrm{T}}$ & 0.0687 & 0.1278 & 0.1151 & 0.7778 & 1.0852 & 0.9433 & $A_{2} \succ A_{3} \succ A_{1}$ & $A_{2}$ \\
0.2 & $(0.29,0.04,0.30,0.18,0.19)^{\mathrm{T}}$ & 0.0584 & 0.1092 & 0.0953 & 0.6385 & 0.9607 & 0.8585 & $A_{2} \succ A_{3} \succ A_{1}$ & $A_{2}$ \\
0.3 & $(0.32,0.04,0.27,0.18,0.18)^{\mathrm{T}}$ & 0.0675 & 0.1209 & 0.1091 & 0.6864 & 1.0077 & 0.9138 & $A_{2} \succ A_{3} \succ A_{1}$ & $A_{2}$ \\
0.4 & $(0.32,0.04,0.27,0.18,0.18)^{\mathrm{T}}$ & 0.0714 & 0.1260 & 0.1151 & 0.7069 & 1.0278 & 0.9375 & $A_{2} \succ A_{3} \succ A_{1}$ & $A_{2}$ \\
0.5 & $(0.32,0.04,0.27,0.18,0.18)^{\mathrm{T}}$ & 0.0649 & 0.1176 & 0.1052 & 0.6727 & 0.9942 & 0.8980 & $A_{2} \succ A_{3} \succ A_{1}$ & $A_{2}$ \\
0.6 & $(0.32,0.04,0.27,0.18,0.18)^{\mathrm{T}}$ & 0.0714 & 0.1260 & 0.1151 & 0.7069 & 1.0278 & 0.9375 & $A_{2} \succ A_{3} \succ A_{1}$ & $A_{2}$ \\
0.7 & $(0.32,0.04,0.27,0.18,0.18)^{\mathrm{T}}$ & 0.0623 & 0.1142 & 0.1012 & 0.6590 & 0.9808 & 0.8822 & $A_{2} \succ A_{3} \succ A_{1}$ & $A_{2}$ \\
0.8 & $(0.30,0.04,0.27,0.20,0.19)^{\mathrm{T}}$ & 0.0710 & 0.1010 & 0.1123 & 0.6568 & 0.9264 & 0.9821 & $A_{3} \succ A_{2} \succ A_{1}$ & $A_{3}$ \\
0.9 & $(0.30,0.04,0.27,0.20,0.19)^{\mathrm{T}}$ & 0.0705 & 0.1012 & 0.1023 & 0.4569 & 1.1020 & 0.8744 & $A_{3} \succ A_{2} \succ A_{1}$ & $A_{3}$ \\
1.0 & $(0.30,0.04,0.27,0.20,0.19)^{\mathrm{T}}$ & 0.0742 & 0.0975 & 0.1103 & 0.7069 & 1.0278 & 0.9375 & $A_{3} \succ A_{2} \succ A_{1}$ & $A_{3}$ \\
\hline
\end{tabular}

It can be seen from Table 7 that, for different risk attitude parameter values $\lambda$, the optimal weight vectors of attributes obtained may be different. If $\lambda \in[0,0.2]$, $\omega^{*}=(0.29,0.04,0.30,0.18,0.19)^{\mathrm{T}} \quad ; \quad$ if $\lambda \in[0.3,0.7]$, 
$\omega^{*}=(0.32,0.04,0.27,0.18,0.18)^{\mathrm{T}} \quad ; \quad$ if $\quad \lambda \in(0.7,1.0] \quad$, $\omega^{*}=(0.30,0.04,0.27,0.20,0.19)^{\mathrm{T}}$. The ranking orders of candidates are also not completely the same. If $\lambda \in[0,0.7]$, the ranking orders of candidates is $A_{2} \succ A_{3} \succ A_{1}$, the best candidate is $A_{2}$; if $\lambda \in[0.8,1.0]$, the ranking orders of candidates is $A_{3} \succ A_{2} \succ A_{1}$, the best candidate is $A_{3}$.

The above analysis suggests that the DM's risk attitude parameter indeed plays an important role in the decision making. Since TIFN is a special kind of intuitionistic fuzzy number, involving DM's risk attitude to rank the TIFNs is very reasonable and necessary. When the risk attitude parameter value is different, the corresponding decision result may be different.

To further research the sensitivity analysis of objective weights or importance $\theta_{1}$ and $\theta_{2}$ in Eq. (46), the different values of $\theta_{1}$ and $\theta_{2}$ are chosen to solve Eq. (46), such as $\theta_{1}=0.0$ and $\theta_{2}=1.0, \theta_{1}=0.1$ and $\theta_{2}=0.9$, $\theta_{1}=0.2$ and $\theta_{2}=0.8, \theta_{1}=0.3$ and $\theta_{2}=0.7, \theta_{1}=0.4$ and $\theta_{2}=0.6, \theta_{1}=0.6$ and $\theta_{2}=0.4$, etc. We can obtain the identical optimal weight vectors of attributes, i.e., $\omega^{*}=(0.32,0.04,0.27,0.18,0.18)^{\mathrm{T}}$. Thus, the ranking orders of candidates are the same, which demonstrates that the optimal weight vectors of attributes and ranking orders of candidates are not sensitive to the objective weights or importance $\theta_{1}$ and $\theta_{2}$ as far as this personnel selection example is concerned.

\subsection{Comparison analysis of the computational results obtained}

In this subsection, we compare the results obtained by the method $\left(\mathrm{Li}^{7}\right)$ and the proposed method in this paper.

(1) The method $\left(\mathrm{Li}^{7}\right)$ and the proposed method in this paper can be used to solve the MADM problems in which the ratings of alternatives on attributes are expressed with TIFNs

$\mathrm{Li}^{7}$ introduced the ratio of the value index to the ambiguity index and applied to MADM problems using TIFNs with the attribute weighs are completely known. In $\mathrm{Li}^{7}$, single DM is considered and the decision matrix of application example is just the above Table 1 of this paper. The ranking order of the three candidates is generated as follows: $A_{1} \succ A_{3} \succ A_{2}$ if $\lambda \in[0,0.1899)$; $A_{3} \succ A_{1} \succ A_{2}$ if $\lambda \in(0.1899,0.9667) ; A_{2} \succ A_{3} \succ A_{1}$ if $\lambda \in(0.9667,1]$.

If we do not consider the DMs $P_{2}$ and $P_{3}$ in the above personnel selection example, namely, only consider DM $P_{1}$ (i.e., $v_{1}=1, v_{2}=0, v_{3}=0$ ) and Table 1, then the above MAGDM problem reduces to the MADM problem. The proposed method in this paper can also be used to solve this MADM problem and the ranking order of the three candidates can be obtained as follows: $A_{2} \succ A_{3} \succ A_{1}$ if $\lambda \in[0,0.7] ; A_{3} \succ A_{2} \succ A_{1}$ if $\lambda \in[0.8,1.0]$. Thus, the ranking order obtained in this paper is different from that obtained in $\mathrm{Li}^{7}$.

(2) In the situation of the attribute weights completely known, the method $\left(\mathrm{Li}^{7}\right)$ and the proposed method in this paper can be used to solve the MADM problems with TIFNs

The proposed method in this paper can compute the ranking indices through directly using Eq. (35) and (36) and easily rank the alternatives. Thus, not only the MADM problems with TIFNs but also the MAGDM problems with TIFNs can be easily solved using the proposed method in this paper, while the method $\left(\mathrm{Li}^{7}\right)$ can only deal with the MADM problems with TIFNs.

(3) In the situation of the attribute weights completely unknown, the method $\left(\mathrm{Li}^{7}\right)$ can not be used to solve the MADM problems with TIFNs whereas the proposed method in this paper is very suitable for this case. Only removing the incomplete weight information structure $\Lambda$ from Eq. (46), the attribute weight vector can be easily obtained by solving Eq. (46). Further, the ranking indices can be calculated by using Eqs. (35) and (36) and then the ranking orders of alternatives can be generated.

(4) In the situation of the incomplete weight information structures provided, the models and method proposed in this paper are applicable to the MADM and MAGDM problems with TIFNs. Solving linear programming (i.e., Eq. (46)) by Simplex Method, the attribute weight vectors can be easily obtained. However, the method $\left(\mathrm{Li}^{7}\right)$ is unable to deal with the MADM and MAGDM problems with TIFNs and weight preference information structures since it is only appropriate for the MADM problems with the completely known attribute weights.

In sum, the research problems and principles of decision making by the method $\left(\mathrm{Li}^{7}\right)$ and the proposed method in this paper are remarkably different. The former researched the MADM problems with TIFNs and completely known weights. The latter researched the MAGDM problems with TIFNs and incomplete weight information structure. The latter can simultaneously determine the attribute weights and generate the ranking order of alternatives through solving the auxiliary linear programming model derived from the multi-objective optimization model. At the same time, the latter can be applicable to the MADM and MAGDM problems in which the weights are completely unknown and the weights are completely known. Therefore, the models and method proposed in 
this paper are more universal and flexible than that proposed in $\mathrm{Li}^{7}$.

\section{Conclusions}

The IFNs are the suitable and strong tools for quantifying an ill-known quantity. The concepts of the possibility mean, variance of TIFNs as well as standard deviation are introduced and then a new ranking method of TIFNs considering the risk attitude of DM is proposed based on the possibility mean and standard deviation. Thus, a new method is developed to solve MAGDM problems with TIFNs and incomplete weight preference information. The comparison analysis shows that the models and method proposed in this paper have some remarkable advantages over other existing methods. Obviously, the models and method proposed in this paper are applicable to lots of similar decision problems although they are illustrated with the example of the personnel selection problem. The critical factor for the application of the proposed method is how to reasonably represent the assessment information with TIFNs, which will be further researched in the near future.

\section{Acknowledgements}

The authors would like to thank the anonymous reviewers for their insightful and constructive comments. This work was partially supported by the National Natural Science Foundation of China (Nos. 71061006, 61263018), the Humanities Social Science Programming Project of Ministry of Education of China (No. 09YGC630107), the Natural Science Foundation of Jiangxi Province of China (No. 20114BAB201012), the Science and Technology Project of Jiangxi province educational department of China (Nos. GJJ12265 and GJJ12740) and the Excellent Young Academic Talent Support Program of Jiangxi University of Finance and Economics.

\section{References}

1. Atanassov, K T. Intuitionistic fuzzy sets. Fuzzy Sets and Systems, 20 (1986) 87-96.

2. Zadeh, L. A. Fuzzy sets. Information and Control, 18 (1965) 338-353.

3. Atanassov, K T., Gargov, G. Interval-valued intuitionistic fuzzy sets. Fuzzy Sets and Systems, 31 (1989) 343-349.

4. Dubois, D., Prade, H. Fuzzy Sets and Systems: Theory and Applications. (Academic Press, New York, 1980).

5. Li, D. F. A note on "using intuitionistic fuzzy sets for faulttree analysis on printed circuit board assembly". Microelectronics Reliability, 48 (2008) 1741.
6. Shu, M. H., Cheng, C. H., Chang, J. R. Using intuitionistic fuzzy sets for fault tree analysis on printed circuit board assembly. Microelectronics Reliability, 46 (2006) 21392148.

7. Li, D. F. A ratio ranking method of triangular intuitionistic fuzzy numbers and its application to MADM problems. Computers and Mathematics with Applications, 60 (2010) 1557-1570.

8. Li, D. F., Nan, J. X., Zhang, M. J. A ranking method of triangular intuitionistic fuzzy numbers and application to decision making. International Journal of Computational Intelligence Systems, 3 (2010) 522-530.

9. Nan, J. X., Li, D. F., Zhang, M. J. A lexicographic method for matrix games with payoffs of triangular intuitionistic fuzzy Numbers. International Journal of Computational Intelligence Systems, 3 (2010) 280-289.

10. Zadeh, L. A. Fuzzy sets as a basis for a theory of possibility. Fuzzy Sets and Systems, 1 (1978) 3-28.

11. Dubois, D., Prade, H. The mean value of a fuzzy number. Fuzzy Sets and Systems, 24 (1987) 279-300.

12. Carlsson, C., Fullér, R. On possibilistic mean value and variance of fuzzy numbers. Fuzzy Sets and Systems, 122 (2001) 315-326.

13. Fullér, R., Majlender, P. On weighted possibilistic mean and variance of fuzzy numbers. Fuzzy Sets and Systems, 136 (2003) 363-374.

14. Saeidifar, A., Pasha, E. The possibilistic moments of fuzzy numbers and their applications. Journal of computational and applied mathematics 223 (2009) 1028-1042.

15. Chen, W., Tan, S. H. On the possibilistic mean value and variance of multiplication of fuzzy numbers. Journal of computational and applied mathematics, 232 (2009) 327334.

16. Carlsson, C., Fullér, R., Majlender, P. A possibilistic approach to selecting portfolios with highest utility score. Fuzzy Sets and Systems, 131 (2002) 13-21.

17. Jana, P., Roy, T. K., Mazumder, S. K. Multi-objective possibilistic model for portfolio selection with transaction cost. Journal of computational and applied mathematics, 228 (2009) 188-196.

18. Zhang, W. G., Xiao, W. L. On weighted lower and upper possibilistic means and variances of fuzzy numbers and its application in decision. Knowledge and Information Systems, 18 (2009) 311-330.

19. Wan, S. P., Li D. F. Possibility mean and variance based method for multi-attribute decision making with triangular intuitionistic fuzzy numbers. Journal of Intelligent and Fuzzy Systems, 24 (2013) 743-754.

20. Wan, S. P., Wang Q. Y., Dong Q. Y., The extended VIKOR method for multi-attribute group decision making with triangular intuitionistic fuzzy numbers. Knowledge-Based Systems, 52 (2013) 65-77.

21. Herrera-Viedma, E., Chiclana, F., Herrera, F., Alonso, S. Group decision-making model with incomplete fuzzy preference relations based on additive consistency. IEEE Transactions on Systems, Man, and Cybernetics-Part B: Cybernetics 1 (2007) 176-189.

22. Podinovski, V. V. Set choice problems with incomplete information about the preferences of the decision makers. European Journal of Operation Research, 207 (2010) 371379. 
23. Wang, Z. J., Li, K. W. Wang, W. Z. An approach to multiattribute decision making with interval-valued intuitionistic fuzzy assessments and incomplete weights. Information Sciences, 179 (2009) 3026-3040.

24. Li, D. F. Closeness coefficient based nonlinear programming method for interval-valued intuitionistic fuzzy multiattribute decision making with incomplete preference information. Applied Soft Computing, 11 (2011) 3402-3418.
25. Wan, S. P., Li, D. F. Fuzzy LINMAP approach to heterogeneous MADM considering the comparisons of alternatives with hesitation degrees. Omega, 41(6) (2013) 925-940.

26. Li, D. F., Wan, S. P. Fuzzy linear programming approach to multiattribute decision making with multiple types of attribute values and incomplete weight information, Applied Soft Computing, 13 (2013) 4333-4348. 